\title{
Multistep Collocation Methods for Volterra Integral Equations with Weakly Singular Kernels
}

\author{
Jingjun Zhao, Teng Long and Yang $\mathrm{Xu}^{*}$ \\ Department of Mathematics, Harbin Institute of Technology, \\ Harbin 150001, China.
}

Received 3 January 2018; Accepted (in revised version) 7 May 2018.

\begin{abstract}
A multistep collocation method on uniform grids for weakly singular Volterra integral equations is considered. The initial integral equation is modified by a smoothing transformation and then solved by a multistep collocation method on a uniform grid. Convergence and linear stability are also studied. Numerical results demonstrate the efficiency of the method.
\end{abstract}

AMS subject classifications: 65L20

Key words: Multistep collocation method, Volterra integral equation, weakly singular kernel.

\section{Introduction}

Let $0<\alpha<1$ and $D:=\{(t, \tau): 0 \leqslant \tau \leqslant t \leqslant T\}$. Linear weakly singular Volterra Integral Equations (VIEs)

$$
y(t)=g(t)+\int_{0}^{t} K(t, \tau) y(\tau) \mathrm{d} \tau, \quad t \in[0, T],
$$

where $K(t, \tau)=(t-\tau)^{-\alpha} k(t, \tau)$, arise in many practical applications, including combustion, viscoelasticity, control theory, finance, biology, optics and so on - cf. [16, 19, 24, 25]. Such equations are well-studied and numerous results for the Eq. (1.1) can be immediately extended on nonlinear VIEs

$$
y(t)=g(t)+\int_{0}^{t}(t-\tau)^{-\alpha} k(t, \tau, y(\tau)) \mathrm{d} \tau, \quad t \in[0, T],
$$

if $k: D \times \mathbb{R} \rightarrow \mathbb{R}$ and $g:[0, T] \rightarrow \mathbb{R}$ are sufficiently smooth functions - cf. [5, 7, 11].

Various numerical methods for weakly singular VIEs have been also developed [10, 18, $20,27]$. In particular, while dealing with weak singularity the collocation methods exploit two main ideas. Thus one can apply standard methods on suitable non-uniform meshes and

*Corresponding author. Email addresses: hit_zjj@hit.edu.cn (Jingjun Zhao), yangx@hit.edu.cn (Yang Xu) 
this approach was used in one-step collocation methods $[5,8,15]$. Nevertheless, the use of small subintervals at the start of the method can lead to substantial round-off errors in subsequent calculations. To overcome these difficulties, various smoothing transformations have been proposed in $[1,2,17,22,23]$. Thus Diogo et al. [16] regularised weakly singular VIEs by a transformation and applied spline collocation methods based on uniform meshes. We note that working with smoothing transformations on a uniform partitions is equivalent to the use of graded meshes.

On the other hand, multistep collocation methods attract a lot of interest nowadays. Such methods employ collocation polynomials, relying on data and collocation points from previous time steps. The use of such methods allows to achieve prescribed accuracy at a much lower computational cost than in classical one-step collocation methods [12]. Moreover, multistep collocation methods have good stability. We recall that two-step almost collocation methods for VIEs with regular kernels have been constructed in $[9,11,12]$, but generalisation of this approach to VIEs with weakly singular kernels requires substantial additional work. Here we engage the transformation from [23] to extend the approach of Conte and Paternoster [12] to weakly singular VIEs.

This paper is arranged as follows. Section 2 is devoted to the regularity of the solutions of the Eq. (1.1). In Section 3, multistep collocation methods for weakly singular VIEs are introduced and the order of convergence of such methods is established. In Section 4, we analyse the stability of multistep methods. Finally, Section 5 presents the results of numerical experiments, which illustrate the theoretical findings.

\section{Regularity of the Solutions}

Here and in what follows, $C$ denotes a positive constant, independent of specified variables but taking different values in different occurrences. Let us introduce the weight function

$$
\Lambda_{\theta}(t):= \begin{cases}1, & \text { for } \theta<0, \\ (1+|\log t|)^{-1}, & \text { for } \theta=0, \\ t^{\theta}, & \text { for } \theta>0,\end{cases}
$$

where $t \in(0, T]$. We also consider two sets of smooth functions needed to describe the high order derivatives of the solutions of the Eq. (1.1).

Definition 2.1 (cf. Refs. $[14,23]$ ). If $m \in \mathbb{N}$ and $\alpha<1$, then $C^{m, \alpha}[0, T]$ is the set of all $m$-times continuously differentiable functions $y:[0, T] \rightarrow \mathbb{R}$ such that

$$
\left|y^{(j)}(t)\right| \leqslant C \Lambda_{\alpha+j-1}^{-1}(t), \quad j=1,2, \cdots, m .
$$

Definition 2.2 (cf. Refs. $[14,23]$ ). If $m \in \mathbb{N}$ and $\alpha<1$, then $W^{m, \alpha}(D)$ is the set of all $m$-times continuously differentiable functions $K: D \rightarrow \mathbb{R}$ such that

$$
\left|\left(\frac{\partial}{\partial t}\right)^{i}\left(\frac{\partial}{\partial t}+\frac{\partial}{\partial \tau}\right)^{j} K(t, \tau)\right| \leqslant C \Lambda_{\alpha+i}^{-1}(t-\tau), \quad i+j \leqslant m, \quad i, j \in \mathbb{N}^{+} .
$$


Lemma 2.1. If $K(t, \tau) \in W^{m, \alpha}(D), m \in \mathbb{N}, \alpha<1$, then

$$
\left|\frac{\partial^{p} K(t, \tau)}{\partial t^{l} \partial \tau^{p-l}}\right| \leqslant C \Lambda_{\alpha+p-l}^{-1}(t-\tau), \quad l \leqslant p \leqslant m .
$$

Proof. It follows immediately from Definition 2.2.

The regularity of the solutions of the Eq. (1.1) can be characterised as follows.

Lemma 2.2 (cf. Brunner \& Houwen [8, p. 1081]). If $K \in W^{m, \alpha}(D), g \in C^{m, \alpha}[0, T], m \in \mathbb{N}$, $\alpha<1$, then (1.1) has a unique solution $y \in C^{m, \alpha}[0, T]$.

Now we assume that $m \in \mathbb{N}, V, T \in \mathbb{R}^{+}, d \geqslant 1$ and consider smoothing transformations $\phi:[0, V] \rightarrow[0, T]$ such that

$$
\begin{gathered}
\phi \in C^{m}[0, V], \\
C_{1} v^{d-1} \leqslant \phi^{\prime}(v) \leqslant C_{2} v^{d-1}, \quad 0 \leqslant v \leqslant V, \\
\left|\phi^{(j)}(v)\right| \leqslant C \begin{cases}v^{d-j}, & 0 \leqslant j \leqslant \min \{d, m\}, \\
1, & j>d,\end{cases}
\end{gathered}
$$

where $C_{2} \geqslant C_{1}>0$ and $C>0$ are constants.

If $v, w \in[0, V]$ and $P:=\{(v, w): 0 \leqslant w \leqslant v \leqslant V\}$, we can replace the variables $t$ and $\tau$ in (1.1) by

$$
\tau=\phi(w), \quad t=\phi(v),
$$

thus rewriting this equation in the form

$$
y^{\phi}(v)=g^{\phi}(v)+\int_{0}^{v} K^{\phi}(v, w) y^{\phi}(w) \mathrm{d} w,
$$

where $g^{\phi}(v)=g(\phi(v)), K^{\phi}(v, w)=K(\phi(v), \phi(w)) \phi^{\prime}(w)$ are known functions and $y^{\phi}(v)$ $=y(\phi(v))$ is the objective function. Let us now describe the properties of the functions in (2.6).

Lemma 2.3. If $K(t, \tau) \in W^{m, \alpha}(D)$ and the transformation $\phi$ satisfies the conditions (2.2)(2.4), then for $p=\sum_{k=1}^{i+j} a_{k}+\sum_{k=1}^{j+1} b_{k}-1$ with $a_{k}, b_{k} \in \mathbb{N}_{0}$, there is a constant $C$ such that

$$
\begin{aligned}
& \left(\frac{\partial}{\partial v}\right)^{i}\left(\frac{\partial}{\partial v}+\frac{\partial}{\partial w}\right)^{j} K^{\phi}(v, w) \\
= & \sum_{l=0}^{p} \sum_{a_{k}, b_{k}} C \prod_{k=1}^{i+j}\left(\phi^{(k)}(v)\right)^{a_{k}} \prod_{k=1}^{j+1}\left(\phi^{(k)}(w)\right)^{b_{k}} \frac{\partial^{p} K(t, \tau)}{\partial t^{l} \partial \tau^{p-l}}
\end{aligned}
$$

where $l \leqslant p \leqslant i+j \leqslant m, 0 \leqslant w \leqslant v \leqslant V$ and $\sum_{k=1}^{i+j} k a_{k}+\sum_{k=1}^{j+1} k b_{k}=i+j+1$.

Proof. It can be proved by induction. 
Lemma 2.4 (cf. Pedas \& Vainikko [23, Lemma 4.2]). If $f$ and $\phi$ satisfy the conditions:

1. $f(t) \in C^{m, \alpha}[0, T]$ for $m \in \mathbb{N}, \alpha, T \in \mathbb{R}, \alpha<1$ and $T>0$,

2. $\phi$ meets the requirements (2.2)-(2.4) with $d \in \mathbb{N}$ if $d \leqslant m$ and with $d \in \mathbb{R}$ if $d>m$, then $f^{\phi}(v)=f(\phi(v)) \in C^{m, \beta}[0, V]$ with $\beta=1-d(1-\alpha)$.

Lemma 2.5. If $K$ and $\phi$ satisfy the conditions:

1. $K(t, \tau) \in W^{m, \alpha}(D)$, where $D=\{(t, \tau): 0 \leqslant \tau \leqslant t \leqslant T\}, m \in \mathbb{N}, \alpha, T \in \mathbb{R}, \alpha<1$ and $T>0$,

2. $\phi$ meets the requirements (2.2)-(2.4), with $d \in \mathbb{N}$ if $d \leqslant m$ and with $d \in \mathbb{R}$ if $d>m$,

then $K^{\phi}(v, w)=K(\phi(v), \phi(w)) \phi^{\prime}(w) \in W^{m, \beta}(P)$ with $\beta=1-d(1-\alpha)$.

Proof. Assume that $K$ and $\phi$ satisfy the above conditions and note that $K^{\phi}(v, w)$ is $m$ times continuously differentiable function in the triangle $0<w \leqslant v \leqslant V$. The inequality (2.7) can now be used, but we have to estimate all terms in its right-hand side. Indeed, it follows from (2.1) that

$$
\begin{aligned}
\left|\frac{\partial^{p} K(t, \tau)}{\partial t^{l} \partial \tau p^{p-l}}\right| & \leqslant C \Lambda_{\alpha+p-l}^{-1}(\phi(v)-\phi(w)) \\
& \leqslant C \begin{cases}1, & \alpha+p-l<0, \\
1+|\log (v-w)|+|\log w|, & \alpha+p-l=0, \\
(v-w)^{-(\alpha+p-l)} w^{-(\alpha+p-l)(d-1)}, & \alpha+p-l>0,\end{cases} \\
& \leqslant C \Lambda_{\alpha+p-l}^{-1}(v-w) .
\end{aligned}
$$

There are five cases to consider — viz.
A: $i \geqslant 1, i+j=1,2, \cdots, \min \{d, m\}$
B: $i \geqslant 1,1+j=1,2, \cdots, d, i+j=d+1, d+2, \cdots, m$;
C: $i \geqslant 1,1+j=d+1, d+2, \cdots, m$;
D: $i=0,1+j=1,2, \cdots, \min \{d, m\}$;
D: $i=0, j=d, d+1, \cdots, m$.

Let us start with the case A. Using the relations (2.1)-(2.5) when $i+j \leqslant \min \{d, m\}$, we obtain

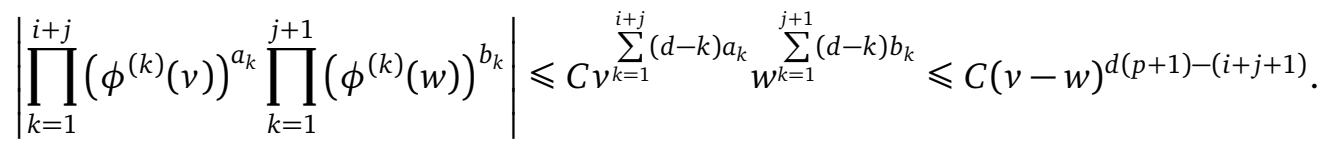


Note that if $0<w \leqslant v \leqslant V$, then $(v-w)^{d(p+1)-(i+j+1)} \leqslant C$. Therefore,

$$
\begin{aligned}
\Psi_{\mathrm{A}} & \leqslant C(v-w)^{d(p+1)-(i+j+1)} \Lambda_{\alpha+p-l}^{-1}(v-w) \\
& \leqslant C \begin{cases}1, & \alpha+p-l<0, \\
(1+|\log (v-w)|)(v-w)^{d(1-\alpha)-(i+1)}, & \alpha+p-l=0, \\
(v-w)^{d(1-\alpha)-(i+1)}, & \alpha+p-l>0,\end{cases}
\end{aligned}
$$

where

$$
\Psi_{\mathrm{A}}=\left|\prod_{k=1}^{i+j}\left(\phi^{(k)}(v)\right)^{a_{k}} \prod_{k=1}^{j+1}\left(\phi^{(k)}(w)\right)^{b_{k}} \frac{\partial^{p} K(t, \tau)}{\partial t^{l} \partial \tau^{p-l}}\right| .
$$

If $\alpha<0$, then $d(1-\alpha)-(i+1)>0$ and the inequality (2.8) yields

$$
\Psi_{\mathrm{A}} \leqslant C .
$$

On the other hand, if $\alpha=0$, then

$$
\Psi_{\mathrm{A}} \leqslant C \begin{cases}1, & i \neq d-1, \\ 1+|\log (v-w)|, & i=d-1 .\end{cases}
$$

For $0<\alpha<1$, one notes that $p>l-\alpha$ and the application of (2.8) shows that

$$
\Psi_{\mathrm{A}} \leqslant C \begin{cases}1, & i \leqslant d(1-\alpha)-1 \\ (v-w)^{d(1-\alpha)-(i+1)}, & i>d(1-\alpha)-1 .\end{cases}
$$

Combining estimates (2.9)-(2.11), we arrive at the inequality

$$
\Psi_{\mathrm{A}} \leqslant C \Lambda_{1+i-d(1-\alpha)}^{-1}(v-w) .
$$

Case B: Let

$$
\begin{aligned}
& Q_{1}=d\left(\sum_{k=1}^{d} a_{k}+\sum_{k=1}^{j+1} b_{k}\right)-\left(\sum_{k=1}^{d} k a_{k}+\sum_{k=1}^{j+1} k b_{k}\right) \geqslant 0, \\
& Q_{2}=d \sum_{k=d+1}^{i+j} a_{k}+\left(\sum_{k=1}^{d} k a_{k}+\sum_{k=1}^{j+1} k b_{k}\right) .
\end{aligned}
$$

It follows from (2.1), (2.2)-(2.4) that

$$
\left|\prod_{k=1}^{i+j}\left(\phi^{(k)}(v)\right)^{a_{k}} \prod_{k=1}^{j+1}\left(\phi^{(k)}(w)\right)^{b_{k}}\right| \leqslant C(v-w)^{Q_{1}},
$$

and since $C(v-w)^{Q_{1}-(\alpha+p-l)} \leqslant C(v-w)^{d(1-\alpha)+j-Q_{2}}$, we obtain

$$
\Psi_{\mathrm{B}} \leqslant C \begin{cases}1, & \alpha+p-l<0 \\ (1+|\log (v-w)|)(v-w)^{Q_{1},}, & \alpha+p-l=0 \\ (v-w)^{d(1-\alpha)+j-Q_{2},} & \alpha+p-l>0\end{cases}
$$


where

$$
\Psi_{\mathrm{B}}=\left|\prod_{k=1}^{i+j}\left(\phi^{(k)}(v)\right)^{a_{k}} \prod_{k=1}^{j+1}\left(\phi^{(k)}(w)\right)^{b_{k}} \frac{\partial^{p} K(t, \tau)}{\partial t^{l} \partial \tau^{p-l}}\right| .
$$

On the other hand, since $C(v-w)^{d(1-\alpha)+j-Q_{2}} \leqslant C(v-w)^{d(1-\alpha)-(i+1)}$, then

$$
\Psi_{\mathrm{B}} \leqslant C\left\{\begin{array}{lll}
1, & \alpha+p-l<0, & \\
1, & \alpha+p-l=0, & Q_{1}>0, \\
1+|\log (v-w)|, & \alpha+p-l=0, & Q_{1}=0, \\
1, & \alpha+p-l>0, & d(1-\alpha)+j-Q_{2} \geqslant 0, \\
(v-w)^{d(1-\alpha)-(i+1)}, & \alpha+p-l>0, & d(1-\alpha)+j-Q_{2}<0 .
\end{array}\right.
$$

The inequality $i+j>d$ implies

$$
Q_{2} \leqslant i+j+1 \text {. }
$$

Moreover, it follows from the relations $\alpha+p-l=0, Q_{1}=0$ and $i+j>d$ that

$$
\begin{aligned}
& i+j+1=d\left(\sum_{k=1}^{d} a_{k}+\sum_{k=1}^{j+1} b_{k}\right)+\sum_{k=d+1}^{i+j} k a_{k}, \\
& d(1-\alpha) \leqslant d(l+1-\alpha)=d(p+1)=d\left(\sum_{k=1}^{i+j} a_{k}+\sum_{k=1}^{j+1} b_{k}\right) \leqslant i+j+1 .
\end{aligned}
$$

However, since $i+j+1 \geqslant d+2$ implies $d(1-\alpha)=i+1$, the inequalities (2.13), (2.14) yield

$$
\Psi_{\mathrm{B}} \leqslant C \Lambda_{1+i-d(1-\alpha)}^{-1}(v-w) .
$$

The remaining cases $\mathbf{C}, \mathbf{D}$ and $\mathbf{E}$, can be considered analogously, so that

$$
\begin{aligned}
& \Psi_{C} \leqslant C \Lambda_{1+i-d(1-\alpha)}^{-1}(v-w), \\
& \Psi_{\mathrm{D}} \leqslant C \Lambda_{1-d(1-\alpha)}^{-1}(v-w), \\
& \Psi_{\mathrm{E}} \leqslant C \Lambda_{1-d(1-\alpha)}^{-1}(v-w) .
\end{aligned}
$$

Combining (2.12), (2.15), (2.16) with the representation (2.7) produces the estimate

$$
\left|\left(\frac{\partial}{\partial v}\right)^{i}\left(\frac{\partial}{\partial v}+\frac{\partial}{\partial w}\right)^{j} K^{\phi}(v, w)\right| \leqslant C \Lambda_{\beta+i}^{-1}(v-w),
$$

where $v, w \in P$ and $i+j \leqslant m$. This shows that $K^{\phi}(v, w) \in W^{m, \beta}(P)$ with $\beta=1-d(1-\alpha)$.

Lemma 2.5 describes the differential properties of the solutions and is used in the convergence analysis below.

Remark 2.1. Simple calculations show that $\beta \leqslant \alpha$, hence the functions $f^{\phi}(v)$ and $K^{\phi}(v, w)$ have improved smoothing properties. 


\section{Convergence Order}

Weakly singular VIEs on graded meshes with grading exponent $\zeta$ are equivalent to VIEs on uniform meshes regularized by transformation $t=v^{\zeta} \mathrm{T}$.

It is well known that the application of linear multistep methods with non-equidistant nodes to ordinary differential equations is connected with a number of problems. For weakly singular VIEs, analogous difficulties arise in multistep collocation methods based on graded meshes. Therefore, here we start with approximation methods on uniform meshes.

Let $N \in \mathbb{N}$ and $V \in \mathbb{R}^{+}$. Following the notation in $[3,4,23]$, we write $\Delta_{N}^{(\zeta, V)}$ for the partition

$$
v_{n}:=V\left(\frac{n}{N}\right)^{\zeta}, \quad n=0,1, \cdots, N, \quad \zeta \geqslant 1,
$$

and discretise the interval $[0, V]$ by uniform mesh $\Delta_{N}^{(1, V)}$ with the step size $h=V / N$. Besides, we consider collocation points $v_{n, j}=v_{n}+c_{j} h$, for $j=1,2, \cdots, m, n=0,1, \cdots, N-1$ with the fixed parameters $0<c_{1}<c_{2}<\cdots<c_{m} \leqslant 1$. Using the transformation $\phi$, we transform the initial mesh into a non-uniform one - viz.

$$
\Delta_{N}^{(\zeta, T)}=\left\{t_{n} \mid t_{n}=\phi\left(v_{n}\right)=T\left(\frac{n}{N}\right)^{\zeta_{n}}, n=0,1, \cdots, N, \zeta=\max _{n=0,1, \cdots, N} \zeta_{n}, \zeta_{n} \geqslant 1\right\} .
$$

Let $t_{n, j}=\phi\left(v_{n, j}\right)=t_{n}+c_{n, j} h_{n}$, where $h_{n}=t_{n+1}-t_{n}$ and

$$
c_{n, j}=\frac{\phi\left(v_{n, j}\right)-\phi\left(v_{n}\right)}{\phi\left(v_{n+1}\right)-\phi\left(v_{n}\right)} .
$$

Using this mesh $\Delta_{N}^{(\zeta, T)}$ in the Eq. (1.1), we rewrite it on the interval $t \in\left[t_{n}, t_{n+1}\right]$ as $y(t)=$ $F_{n}(t)+\Phi_{n}(t)$, where the lag-term $F_{n}(t)$ and the increment-term $\Phi_{n}(t)$ have the form

$$
\begin{aligned}
& F_{n}(t)=g(t)+\int_{0}^{t_{n}} K(t, \tau) y(\tau) \mathrm{d} \tau \\
& \Phi_{n}(t)=\left(\frac{t-t_{n}}{2}\right)^{1-\alpha} \int_{-1}^{1}(1-s)^{-\alpha} k\left(t, t_{n}+\frac{t-t_{n}}{2}(1+s)\right) y\left(t_{n}+\frac{t-t_{n}}{2}(1+s)\right) \mathrm{d} s .
\end{aligned}
$$

If $m$ is a non-negative integer and $-1 \leqslant \vartheta \leqslant m-1$, then $S_{m}^{(\vartheta)}\left(\Delta_{N}^{(\zeta, T)}\right)$ refers to the spline space

$$
f \in C^{\vartheta}([0, T]),\left.\quad f\right|_{\left(t_{n}, t_{n+1}\right]}=f_{n} \in \pi_{m}, \quad n=0,1, \cdots, N-1
$$

on the grid $\Delta_{N}^{(\zeta, T)}$, where $\pi_{m}$ denote the set of (real) polynomials of degree at most $m$. For $N, m \in \mathbb{N}$, the desired approximation of $y^{\phi}$ is an element $u^{\phi}(v) \in S_{m-1}^{(-1)}\left(\Delta_{N}^{(1, V)}\right)$ and $y_{N}(t):=u^{\phi}\left(\phi^{-1}(t)\right), t \in[0, T]$ represents an approximation for the solution $y$ of (1.1). In order to derive the starting values of the scheme, we assume that the domain can be appropriately extended into negative half-axis. The initial values we need, can be obtained via a starting procedure related to a classical one-step method. In this way, the multistep 
collocation method can start on the first integration interval. In what follows, the starting interval is written as $\left[0, v_{r-1}\right]$ and on the set $\left[v_{n}, v_{n+1}\right], n=r-1, r, \cdots, N-1$, the unknown function $y^{\phi}(v)$ is approximated by piecewise polynomials as follows

$$
u_{n}^{\phi}\left(v_{n}+s h\right)=\sum_{k=0}^{r-1} \varphi_{k}(s) y_{n-k}^{\phi}+\sum_{j=1}^{m} \psi_{j}(s) U_{n, j}^{\phi}, \quad s \in[0,1]
$$

Note that $U_{n, j}^{\phi}=u_{n}^{\phi}\left(v_{n, j}\right)$ and $\varphi_{k}(s), \psi_{j}(s)$ are polynomials of degree $m+r-1$, satisfying the conditions $u_{n}^{\phi}\left(v_{n-k}\right)=y_{n-k}^{\phi}$. In particular, the interpolation conditions yield

$$
\begin{aligned}
& \varphi_{l}(-k)=\delta_{l, k}, \quad \varphi_{l}\left(c_{j}\right)=0, \quad l, k=0,1, \cdots, r-1, \\
& \psi_{i}(-k)=0, \quad \psi_{i}\left(c_{j}\right)=\delta_{i, j}, \quad i, j=1,2, \cdots, m,
\end{aligned}
$$

where $\delta_{i, j}$ is the Kronecker delta. The above system has a unique solution — viz.

$$
\varphi_{k}(s)=\prod_{i=1}^{m} \frac{s-c_{i}}{-k-c_{i}} \prod_{i=0, i \neq k}^{r-1} \frac{s+i}{-k+i}, \quad \psi_{j}(s)=\prod_{i=0}^{r-1} \frac{s+i}{c_{j}+i} \prod_{i=1, i \neq j}^{m} \frac{s-c_{i}}{c_{j}-c_{i}} .
$$

On the other hand, the collocation polynomials on the interval $\left[t_{n}, t_{n+1}\right]$ have the form

$$
u_{n}\left(t_{n}+s h_{n}\right)=\sum_{k=0}^{r-1} M_{n, k}(s) y_{n-k}+\sum_{j=1}^{m} L_{n, j}(s) U_{n, j}, \quad s \in[0,1], \quad n=0,1, \cdots, N-1,
$$

where $U_{n, j}=u_{n}\left(t_{n, j}\right)$ and $M_{n, k}(s), L_{n, j}(s)$ are polynomials such that

$$
\begin{aligned}
& M_{n, l}\left(-d_{n, k}\right)=\delta_{l, k}, \quad M_{n, l}\left(c_{n, j}\right)=0, \quad l, k=0,1, \cdots, r-1, \\
& L_{n, i}\left(-d_{n, k}\right)=0, \quad L_{n, i}\left(c_{n, j}\right)=\delta_{i, j}, \quad i, j=1,2, \cdots, m,
\end{aligned}
$$

with $d_{n, k}=\left(t_{n}-t_{n-k}\right) / h_{n}$. The unique solution of the above interpolation problem is

$$
M_{n, k}(s)=\prod_{i=1}^{m} \frac{s-c_{n, i}}{-d_{n, k}-c_{n, i}} \prod_{i=0, i \neq k}^{r-1} \frac{s+d_{n, i}}{-d_{n, k}+d_{n, i}}, \quad L_{n, j}(s)=\prod_{i=0}^{r-1} \frac{s+d_{n, i}}{c_{n, j}+d_{n, i}} \prod_{i=1, i \neq j}^{m} \frac{s-c_{n, i}}{c_{n, j}-c_{n, i}} .
$$

Using the transformation (2.5) in the Eq. (3.1) yields

$$
\begin{aligned}
F_{n}^{\phi}(v)= & g^{\phi}(v)+\int_{0}^{v_{n}} K^{\phi}(v, w) y^{\phi}(w) \mathrm{d} w \\
\Phi_{n}^{\phi}(v)= & \left(\frac{\phi(v)-\phi\left(v_{n}\right)}{2}\right)^{1-\alpha} \int_{-1}^{1}(1-s)^{-\alpha} k\left(\phi(v), \phi\left(v_{n}\right)+\frac{\phi(v)-\phi\left(v_{n}\right)}{2}(1+s)\right) \\
& \times y\left(\phi\left(v_{n}\right)+\frac{\phi(v)-\phi\left(v_{n}\right)}{2}(1+s)\right) \mathrm{d} s .
\end{aligned}
$$


It is clear that $y^{\phi}(v)=y(t), g^{\phi}(v)=g(t), F_{n}^{\phi}(v)=F_{n}(t)$ and the Eq. (1.1) transforms into $y^{\phi}(v)=F_{n}^{\phi}(v)+\Phi_{n}^{\phi}(v)$. Thus, the multistep collocation method can be now written as

$$
\begin{aligned}
& U_{n, i}^{\phi}=F_{n, i}^{\phi}+\Phi_{n, i}^{\phi}, \\
& y_{n+1}^{\phi}=\sum_{k=0}^{r-1} \varphi_{k}(1) y_{n-k}^{\phi}+\sum_{j=1}^{m} \psi_{j}(1) U_{n, j}^{\phi},
\end{aligned}
$$

where

$$
\begin{aligned}
F_{n, i}^{\phi}= & g^{\phi}\left(v_{n, i}\right)+h \sum_{l=0}^{n-1} \int_{0}^{1} K^{\phi}\left(v_{n, i}, v_{l}+s h\right) u_{l}^{\phi}\left(v_{l}+s h\right) \mathrm{d} s, \\
\Phi_{n, i}^{\phi}= & \left(\frac{\phi\left(v_{n, i}\right)-\phi\left(v_{n}\right)}{2}\right)^{1-\alpha} \\
& \times \int_{-1}^{1}(1-s)^{-\alpha} k\left(\phi\left(v_{n, i}\right), \phi\left(v_{n}\right)+\frac{\phi\left(v_{n, i}\right)-\phi\left(v_{n}\right)}{2}(1+s)\right) \\
& \times u_{n}\left(\phi\left(v_{n}\right)+\frac{\phi\left(v_{n, i}\right)-\phi\left(v_{n}\right)}{2}(1+s)\right) \mathrm{d} s, \quad i=1,2, \cdots, m .
\end{aligned}
$$

Let us point out that the collocation polynomials (3.2) and (3.3) are aimed to manage the terms (3.6) and (3.7).

We now consider the errors of the approximations (3.2)-(3.5). Let $L(f)$ refer to the linear functional on the space $C^{m, \alpha}[a, b]$ defined by

$$
L(f)=\sum_{l=1}^{m-1}\left(\int_{a}^{b} \mu_{l}(x) f^{(l)}(x) \mathrm{d} x+\sum_{i=1}^{j_{l}} \chi_{i, l} f^{(l)}\left(x_{i, l}\right)\right),
$$

where $\mu_{l}(x)$ are piecewise continuous functions on the interval $[a, b], x_{i, l} \in[a, b]$ and $\chi_{i, l} \in \mathbb{R}^{*}$. Similar to [13, Theorem 3.7.1], we can establish the following result.

Theorem 3.1. If $L(v)=0$ for all $v \in \pi_{m-1}$, then for all $f \in C^{m, \alpha}[a, b]$ one has

$$
L(f)=\int_{a}^{b} f^{(m)}(t) K(t) \mathrm{d} t
$$

where $K(t)=\frac{1}{(m-1) !} L_{x}\left[(x-t)_{+}^{m-1}\right]$ and $L_{x}\left[(x-t)_{+}^{m-1}\right]$ means that $L$ acts on $(x-t)_{+}^{m-1}$ considered as the function of $x$ such that

$$
(x-t)_{+}^{m-1}= \begin{cases}(x-t)^{m-1}, & \text { for } x \geqslant t, \\ 0, & \text { for } x<t .\end{cases}
$$

Proof. Taking into account the representation (3.8) and applying $L$ to the $m$-term Taylor's expansion of $f(x)$ with the remainder in integral form, we have

$$
L(f)=\frac{1}{(m-1) !} \int_{a}^{b} f^{(m)}(t) L_{x}\left[(x-t)_{+}^{m-1}\right] \mathrm{d} t,
$$


since $L(p)=0$ for any $p \in \pi_{m-1}$. This completes the proof.

Lemma 3.1. If $K \in W^{m+r, \alpha}(D)$ and $g \in C^{m+r, \alpha}[0, T]$, then for any $s \in[0,1]$ and distinct collocation abscissas $\left\{c_{j}\right\}_{j=1}^{m}$, the exact solution $y(t)$ of (1.1) satisfies the equations

$$
\begin{aligned}
y^{\phi}\left(v_{n}+s h\right) & =\sum_{k=0}^{r-1} \varphi_{k}(s) y^{\phi}\left(v_{n-k}\right)+\sum_{j=1}^{m} \psi_{j}(s) y^{\phi}\left(v_{n, j}\right)+h^{m+r} R_{m, r, n}^{\phi}(s), \\
y\left(t_{n}+s h_{n}\right) & =\sum_{k=0}^{r-1} M_{n, k}(s) y\left(t_{n-k}\right)+\sum_{j=1}^{m} L_{n, j}(s) y\left(t_{n, j}\right)+h_{n}^{m+r} R_{m, r, n}(s),
\end{aligned}
$$

where

$$
\begin{aligned}
R_{m, r, n}^{\phi}(s) & =\int_{-r+1}^{1} K_{m, r}^{\phi}(s, \sigma)\left[y^{\phi}\left(v_{n}+\sigma h\right)\right]^{(m+r)} \mathrm{d} \sigma \\
R_{m, r, n}(s) & =\int_{-d_{n, r-1}}^{1} K_{m, r}(s, \sigma) y^{(m+r)}\left(t_{n}+\sigma h_{n}\right) \mathrm{d} \sigma \\
K_{m, r}^{\phi}(s, \sigma) & =\frac{(s-\sigma)_{+}^{m+r-1}-\sum_{k=0}^{r-1} \varphi_{k}(s)(-k-\sigma)_{+}^{m+r-1}-\sum_{j=1}^{m} \psi_{j}(s)\left(c_{j}-\sigma\right)_{+}^{m+r-1}}{(m+r-1) !}, \\
K_{m, r}(s, \sigma) & =\frac{(s-\sigma)_{+}^{m+r-1}-\sum_{k=0}^{r-1} M_{n, k}(s)\left(-d_{n, k}-\sigma\right)_{+}^{m+r-1}-\sum_{j=1}^{m} L_{n, j}(s)\left(c_{n, j}-\sigma\right)_{+}^{m+r-1}}{(m+r-1) !} .
\end{aligned}
$$

Proof. If $K \in W^{m+r, \alpha}(D)$ and $g \in C^{m+r, \alpha}[0, T]$, then Lemma 2.2 shows that $y \in$ $C^{m+r, \alpha}[0, T]$. Therefore, Lemmas 2.4 and 2.5 yield the inclusions $g^{\phi} \in C^{m+r, \beta}[0, V]$, $y^{\phi} \in C^{m+r, \beta}[0, V], K^{\phi} \in W^{m+r, \beta}(P), \beta=1-d(1-\alpha)$.

From (3.9), we obtain

$$
\begin{aligned}
& R_{m, r, n}^{\phi}(s)=\frac{1}{h^{m+r}}\left(y^{\phi}\left(v_{n}+s h\right)-\sum_{k=0}^{r-1} \varphi_{k}(s) y^{\phi}\left(v_{n-k}\right)-\sum_{j=1}^{m} \psi_{j}(s) y^{\phi}\left(v_{n, j}\right)\right), \\
& R_{m, r, n}(s)=\frac{1}{h_{n}^{m+r}}\left(y\left(t_{n}+s h_{n}\right)-\sum_{k=0}^{r-1} M_{n, k}(s) y\left(t_{n-k}\right)-\sum_{j=1}^{m} L_{n, j}(s) y\left(t_{n, j}\right)\right) .
\end{aligned}
$$

Replacing both the sets $v_{n}+s h, v_{n-k}, v_{n, j}$ and $t_{n}+s h_{n}, t_{n-k}, t_{n, j}$ into $x$, respectively, leads to the representations

$$
\begin{aligned}
\bar{K}_{m, r}^{\phi}(s, \sigma) & =\frac{1}{(m+r-1) !} R_{m, r, n}^{\phi(x)}\left[\left(x-\left(v_{n}+\sigma h\right)\right)_{+}^{m+r-1}\right] \\
& =\frac{1}{(m+r-1) ! h}\left\{(s-\sigma)_{+}^{m+r-1}-\sum_{k=0}^{r-1} \varphi_{k}(s)(-k-\sigma)_{+}^{m+r-1}\right.
\end{aligned}
$$


Multistep Collocation Methods for VIEs with Weakly Singular Kernels

$$
\begin{aligned}
& \left.-\sum_{j=1}^{m} \psi_{j}(s)\left(c_{j}-\sigma\right)_{+}^{m+r-1}\right\} \\
\bar{K}_{m, r}(s, \sigma)= & \frac{1}{(m+r-1) !} R_{m, r, n}^{(x)}\left[\left(x-\left(t_{n}+\sigma h_{n}\right)\right)_{+}^{m+r-1}\right] \\
= & \frac{1}{(m+r-1) ! h_{n}}\left\{(s-\sigma)_{+}^{m+r-1}-\sum_{k=0}^{r-1} M_{n, k}(s)\left(-d_{n, k}-\sigma\right)_{+}^{m+r-1}\right. \\
& \left.-\sum_{j=1}^{m} L_{n, j}(s)\left(c_{n, j}-\sigma\right)_{+}^{m+r-1}\right\}
\end{aligned}
$$

suggesting that $\bar{K}_{m, r}^{\phi}$ and $\bar{K}_{m, r}$ are Peano kernels for the operators $R_{m, r, n}^{\phi}$ and $R_{m, r, n}$. By Theorem 3.1, we have

$$
\begin{aligned}
& R_{m, r, n}^{\phi}(s)=\int_{-r+1}^{1} h \bar{K}_{m, r}^{\phi}(s, \sigma)\left[y^{\phi}\left(v_{n}+\sigma h\right)\right]^{(m+r)} \mathrm{d} \sigma, \\
& R_{m, r, n}(s)=\int_{-d_{n, r-1}}^{1} h_{n} \bar{K}_{m, r}(s, \sigma) y^{(m+r)}\left(t_{n}+\sigma h_{n}\right) \mathrm{d} \sigma .
\end{aligned}
$$

Setting $K_{m, r}^{\phi}(s, \sigma)=h \bar{K}_{m, r}^{\phi}(s, \sigma)$ if $s \in[-r+1,1]$ and $K_{m, r}(s, \sigma)=h_{n} \bar{K}_{m, r}(s, \sigma)$ if $s \in$ $\left[-d_{n, r-1}, 1\right]$, we complete the proof.

Lemma 3.1 allows to evaluate the error $\varepsilon(v)=y^{\phi}(v)-u^{\phi}(v)$ of the numerical scheme (3.5).

Theorem 3.2. Assume that $K \in W^{m+r, \alpha}(D), g \in C^{m+r, \alpha}[0, T]$ and $\phi$ is a transformation satisfying the conditions (2.2)-(2.4) with $d \in \mathbb{N}$ for $d \leqslant m$ and $d \in \mathbb{R}$ for $d>m$. If, in addition, the starting error satisfies the relation

$$
\|\varepsilon\|_{\infty,\left[0, v_{r-1}\right]}=\mathscr{O}\left(h^{m+r}\right), \quad h \rightarrow 0,
$$

and the spectral radius $\rho(\mathrm{A})$ of the matrix

$$
\mathbf{A}=\left[\begin{array}{cc}
\mathbf{0}_{r-1,1} & \mathbf{I}_{r-1} \\
\varphi_{r-1}(1) & \varphi_{r-2}(1), \cdots, \varphi_{0}(1)
\end{array}\right]
$$

is less than one, then

$$
\|\varepsilon\|_{\infty}=\mathscr{O}\left(h^{m+r-\alpha}\right), \quad h \rightarrow 0 .
$$

Proof. Let $s \in[0,1]$. It follows from Lemma 3.1 that

$$
\begin{aligned}
y^{\phi}\left(v_{n}+s h\right) & =\sum_{k=0}^{r-1} \varphi_{k}(s) y^{\phi}\left(v_{n-k}\right)+\sum_{j=1}^{m} \psi_{j}(s) y^{\phi}\left(v_{n, j}\right)+h^{m+r} R_{m, r, n}^{\phi}(s), \\
y\left(t_{n}+s h_{n}\right) & =\sum_{k=0}^{r-1} M_{n, k}(s) y\left(t_{n-k}\right)+\sum_{j=1}^{m} L_{n, j}(s) y\left(t_{n, j}\right)+h_{n}^{m+r} R_{m, r, n}(s) .
\end{aligned}
$$


Subtracting (3.10) from (3.2) and (3.11) from (3.3), we obtain

$$
\begin{aligned}
\varepsilon\left(v_{n}+s h\right) & =\sum_{k=0}^{r-1} \varphi_{k}(s) \varepsilon_{n-k}+\sum_{j=1}^{m} \psi_{j}(s) \varepsilon_{n, j}+h^{m+r} R_{m, r, n}^{\phi}(s), \quad n \geqslant r-1, \\
\varepsilon\left(t_{n}+s h_{n}\right) & =\sum_{k=0}^{r-1} M_{n, k}(s) \varepsilon_{n-k}+\sum_{j=1}^{m} L_{n, j}(s) \varepsilon_{n, j}+h_{n}^{m+r} R_{m, r, n}(s), \quad n \geqslant r-1,
\end{aligned}
$$

where $\varepsilon_{n-k}=\varepsilon\left(v_{n-k}\right)=\varepsilon\left(t_{n-k}\right), \varepsilon_{n, j}=\varepsilon\left(v_{n, j}\right)=\varepsilon\left(t_{n, j}\right)$. Evaluating the Eqs. (3.4) at $v=v_{n, i}$ yields

$$
\begin{aligned}
F_{n}^{\phi}\left(v_{n, i}\right)= & g^{\phi}\left(v_{n, i}\right)+h \sum_{l=0}^{n-1} \int_{0}^{1} K^{\phi}\left(v_{n, i}, v_{l}+s h\right) y^{\phi}\left(v_{l}+s h\right) \mathrm{d} s, \\
\Phi_{n}^{\phi}\left(v_{n, i}\right)= & \left(\frac{\phi\left(v_{n, i}\right)-\phi\left(v_{n}\right)}{2}\right)^{1-\alpha} \int_{-1}^{1}(1-s)^{-\alpha} \\
& \times k\left(\phi\left(v_{n, i}\right), \phi\left(v_{n}\right)+\frac{\phi\left(v_{n, i}\right)-\phi\left(v_{n}\right)}{2}(1+s)\right) \\
& \times y\left(\phi\left(v_{n}\right)+\frac{\phi\left(v_{n, i}\right)-\phi\left(v_{n}\right)}{2}(1+s)\right) \mathrm{d} s, \quad i=1,2, \cdots, m .
\end{aligned}
$$

Subtracting (3.6) and (3.7) from (3.14), we get

$$
\begin{aligned}
\varepsilon_{n, i}= & h \sum_{l=0}^{n-1} \int_{0}^{1} K^{\phi}\left(v_{n, i}, v_{l}+s h\right) \varepsilon\left(v_{l}+s h\right) \mathrm{d} s+\left(\frac{c_{n, i} h_{n}}{2}\right)^{1-\alpha} \int_{-1}^{1}(1-s)^{-\alpha} \\
& \times k\left(t_{n, i}, t_{n}+\frac{c_{n, i} h_{n}}{2}(1+s)\right) \varepsilon\left(t_{n}+\frac{c_{n, i} h_{n}}{2}(1+s)\right) \mathrm{d} s .
\end{aligned}
$$

The condition (2.2) implies that

$$
\varepsilon\left(v_{l}+s h\right)=h^{m+r} q_{l}(s), \quad l=0,1, \cdots, r-1, \quad s \in[0,1]
$$

with $\left\|q_{l}\right\|_{\infty} \leqslant C$. Substituting (3.12), (3.13) and (3.16) in (3.15) leads to the equation

$$
\begin{aligned}
\epsilon_{n}^{(2)}-h_{n}^{1-\alpha} \tilde{\mathbf{B}}_{n} \epsilon_{n}^{(2)}= & h \sum_{l=r}^{n-1} \tilde{\mathbf{B}}_{n}^{(l)} \epsilon_{l}^{(2)}+h \sum_{l=r}^{n-1} \overline{\mathbf{B}}_{n}^{(l)} \epsilon_{l}^{(1)}+h_{n}^{1-\alpha} \overline{\mathbf{B}}_{n} \epsilon_{n}^{(1)} \\
& +h^{m+r+1} \sum_{l=0}^{n-1} \bar{\rho}_{n}^{(l)}+h_{n}^{m+r+1-\alpha} \bar{\rho}_{n}^{(n)}, \quad n \geqslant r-1,
\end{aligned}
$$

where the terms $\epsilon_{l}^{(1)} \in \mathbb{R}^{r}, \epsilon_{l}^{(2)}, \bar{\rho}_{n}^{(l)} \in \mathbb{R}^{m}, \overline{\mathbf{B}}_{n}^{(l)} \in \mathbb{R}^{m \times r}$ and $\tilde{\mathbf{B}}_{n}, \tilde{\mathbf{B}}_{n}^{(l)} \in \mathbb{R}^{m \times m}$ have the form

$$
\epsilon_{l}^{(1)}=\left[\varepsilon_{l-r+1}, \varepsilon_{l-r+2}, \cdots, \varepsilon_{l}\right]^{\mathrm{T}}, \quad \epsilon_{l}^{(2)}=\left[\varepsilon_{l, 1}, \varepsilon_{l, 2}, \cdots, \varepsilon_{l, m}\right]^{\mathrm{T}},
$$




$$
\begin{aligned}
\left(\overline{\mathbf{B}}_{n}^{(l)}\right)_{i k} & =\int_{0}^{1} K^{\phi}\left(v_{n, i}, v_{l}+s h\right) \varphi_{k}(s) \mathrm{d} s, \quad\left(\tilde{\mathbf{B}}_{n}^{(l)}\right)_{i j}=\int_{0}^{1} K^{\phi}\left(v_{n, i}, v_{l}+s h\right) \psi_{j}(s) \mathrm{d} s, \\
\left(\overline{\mathbf{B}}_{n}\right)_{i k} & =\left(\frac{c_{n, i}}{2}\right)^{1-\alpha} \int_{-1}^{1}(1-s)^{-\alpha} k\left(t_{n, i}, t_{n}+\frac{c_{n, i} h_{n}}{2}(1+s)\right) M_{n, k}\left(\frac{c_{n, i}}{2}(1+s)\right) \mathrm{d} s, \\
\left(\tilde{\mathbf{B}}_{n}\right)_{i j} & =\left(\frac{c_{n, i}}{2}\right)^{1-\alpha} \int_{-1}^{1}(1-s)^{-\alpha} k\left(t_{n, i}, t_{n}+\frac{c_{n, i} h_{n}}{2}(1+s)\right) L_{n, j}\left(\frac{c_{n, i}}{2}(1+s)\right) \mathrm{d} s, \\
\left(\bar{\rho}_{n}^{(l)}\right)_{i} & =\left\{\begin{array}{l}
\int_{0}^{1} K^{\phi}\left(v_{n, i}, v_{l}+s h\right) q_{l}(s) \mathrm{d} s, \quad l=0,1, \cdots, r-1, \\
\int_{0}^{1} K^{\phi}\left(v_{n, i}, v_{l}+s h\right) R_{m, r, l}^{\phi}(s) \mathrm{d} s, \quad l=r, r+1, \cdots, n-1, \\
\left(\frac{c_{n, i}}{2}\right)^{1-\alpha} \int_{-1}^{1}(1-s)^{-\alpha} k\left(t_{n, i}, t_{n}+\frac{c_{n, i} h_{n}}{2}(1+s)\right) R_{m, r, n}\left(\frac{c_{n, i}}{2}(1+s)\right) \mathrm{d} s, \quad l=n .
\end{array}\right.
\end{aligned}
$$

Setting $n=l-1$ and $s=1$ in (3.12) produces the difference equation

$$
\epsilon_{l}^{(1)}=\mathbf{A} \epsilon_{l-1}^{(1)}+\mathbf{S} \epsilon_{l-1}^{(2)}+h^{m+r} \tilde{\rho}_{m, r, l-1}, \quad l \geqslant r-1,
$$

with the coefficients

$$
\begin{aligned}
& \mathbf{S}=\left[\begin{array}{c}
\mathbf{0}_{r-1, m} \\
\boldsymbol{\psi}^{\mathrm{T}}(1)
\end{array}\right], \quad \tilde{\rho}_{m, r, j}=\left[\begin{array}{c}
\mathbf{0}_{r-1,1} \\
R_{m, r, j}^{\phi}(1)
\end{array}\right], \\
& \boldsymbol{\psi}(1)=\left[\psi_{1}(1), \psi_{2}(1), \cdots, \psi_{m}(1)\right]^{\mathrm{T}} .
\end{aligned}
$$

We note that (3.18) has the solution

$$
\epsilon_{l}^{(1)}=\mathbf{A}^{l-r+1} \epsilon_{r-1}^{(1)}+\sum_{j=r-1}^{l-1} \mathbf{A}^{l-j-1}\left(\mathbf{S} \epsilon_{j}^{(2)}+h^{m+r} \tilde{\rho}_{m, r, j}\right),
$$

and inserting it in (3.17), one obtains

$$
\begin{aligned}
& \left(I-h_{n}^{1-\alpha} \tilde{\mathbf{B}}_{n}\right) \epsilon_{n}^{(2)}=h \sum_{l=r}^{n-1} \tilde{\mathbf{B}}_{n}^{(l)} \epsilon_{l}^{(2)}+h\left(\sum_{l=r}^{n-1} \overline{\mathbf{B}}_{n}^{(l)} \mathbf{A}^{l-r+1}\right) \epsilon_{r-1}^{(1)} \\
& +h \sum_{j=r}^{n-2}\left(\sum_{l=j+1}^{n-1} \overline{\mathbf{B}}_{n}^{(l)} \mathbf{A}^{l-j-1} \mathbf{S}\right) \epsilon_{j}^{(2)}+h^{m+r+1} \sum_{j=r-1}^{n-2}\left(\sum_{l=j+1}^{n-1} \overline{\mathbf{B}}_{n}^{(l)} \mathbf{A}^{l-j-1}\right) \tilde{\rho}_{m, r, j} \\
& +h\left(\sum_{l=r}^{n-1} \overline{\mathbf{B}}_{n}^{(l)} \mathbf{A}^{l-r} \mathbf{S}\right) \epsilon_{r-1}^{(2)}+h^{m+r+1} \sum_{l=0}^{n-1} \bar{\rho}_{n}^{(l)}+h_{n}^{1-\alpha} \overline{\mathbf{B}}_{n} \mathbf{A}^{n-r+1} \epsilon_{r-1}^{(1)} \\
& +h_{n}^{1-\alpha}\left(\sum_{j=r-1}^{n-1} \overline{\mathbf{B}}_{n} \mathbf{A}^{n-j-1} \mathbf{S}\right) \epsilon_{j}^{(2)}+h_{n}^{1-\alpha} h^{m+r} \sum_{j=r-1}^{n-1} \overline{\mathbf{B}}_{n} \mathbf{A}^{n-j-1} \tilde{\rho}_{m, r, j}+h_{n}^{m+r+1-\alpha} \bar{\rho}_{n}^{(n)} .
\end{aligned}
$$

The error term $\epsilon_{n}^{(2)}$ can be estimated analogously to [12, Theorem 4.2]. This finishes the proof.

Table 1 shows the convergence order for various approximation methods for weakly singular VIEs. 
Table 1: Collocation methods for weakly singular VIEs

\begin{tabular}{||lcc||}
\hline Method & Collocation numbers & Convergence Order \\
\hline One-step (uniform) & $m$ & $1-\alpha$ \\
One-step (graded) & $m$ & $m$ \\
One-step (transformed) & $m$ & $m$ \\
Multistep (uniform) & $m$ & $1-\alpha$ \\
Multistep (transformed) & $m$ & $m+r-\alpha$ \\
\hline
\end{tabular}

\section{Linear Stability Analysis}

In this section, we consider the basic test equation

$$
y(t)=1+\lambda \int_{0}^{t}(t-\tau)^{-\alpha} y(\tau) \mathrm{d} \tau, \quad 0<\alpha<1, \quad t \in[0, T], \quad \operatorname{Re}(\lambda)<0
$$

usually adopted in literature to test the stability of numerical methods for VIEs- [6]. If $|\arg \lambda-\pi|<(1-\alpha / 2) \pi$, then the exact solution $y(t)$ of (4.1) tends to zero as $t$ tends to $+\infty$.

Definition 4.1. A numerical method is called stable for $z=\lambda h \in \mathbb{C}$, where $h$ is the step size, if the corresponding numerical solution $y_{n}$ of the Eq. (4.1) tends to zero when $n$ tends to $+\infty$. The region of absolute stability of a numerical method consists of all those $z \in \mathbb{C}$ for which the method is stable. If the region of absolute stability contains the left complex half-plane, then the corresponding method is called $A$-stable.

Using the mesh $\Delta_{N}^{(\zeta, T)}$, we rewrite (4.1) on the interval $\left[t_{n}, t_{n+1}\right]$ as

$$
y(t)=F_{n}(t)+\Phi_{n}(t), \quad t \in\left[t_{n}, t_{n+1}\right],
$$

where

$$
\begin{aligned}
& F_{n}(t)=1+\lambda \int_{0}^{t_{n}}(t-\tau)^{-\alpha} y(\tau) \mathrm{d} \tau \\
& \Phi_{n}(t)=\lambda\left(\frac{t-t_{n}}{2}\right)^{1-\alpha} \int_{-1}^{1}(1-s)^{-\alpha} y\left(t_{n}+\frac{t-t_{n}}{2}(1+s)\right) \mathrm{d} s,
\end{aligned}
$$

and the application of $\phi$ yields

$$
\begin{aligned}
& F_{n}^{\phi}(v)=1+\lambda \int_{0}^{v_{n}}(\phi(v)-\phi(w))^{-\alpha} \phi^{\prime}(w) y^{\phi}(w) \mathrm{d} w \\
& \Phi_{n}^{\phi}(v)=\lambda\left(\frac{\phi(v)-\phi\left(v_{n}\right)}{2}\right)^{1-\alpha} \int_{-1}^{1}(1-s)^{-\alpha} y\left(\phi\left(v_{n}\right)+\frac{\phi(v)-\phi\left(v_{n}\right)}{2}(1+s)\right) \mathrm{d} s .
\end{aligned}
$$


Consider now the vectors

$$
\begin{aligned}
& \mathbf{u}=[1,1, \cdots, 1]^{\mathrm{T}} \in \mathbb{R}^{m}, \quad \mathbf{U}_{n}^{\phi}=\left[U_{n, 1}^{\phi}, U_{n, 2}^{\phi}, \cdots, U_{n, m}^{\phi}\right]^{\mathrm{T}}, \\
& \boldsymbol{y}_{n}^{\phi(r)}=\left[y_{n}^{\phi}, y_{n-1}^{\phi}, \cdots, y_{n-r+1}^{\phi}\right]^{\mathrm{T}}, \quad \varphi(1)=\left[\varphi_{0}(1), \varphi_{1}(1), \cdots, \varphi_{r-1}(1)\right]^{\mathrm{T}},
\end{aligned}
$$

and the matrices

$$
\mathbf{E}=\left[\begin{array}{c}
-\boldsymbol{\psi}(1)^{\mathrm{T}} \\
\mathbf{0}_{r, m}
\end{array}\right], \quad \mathbf{F}=\left[\begin{array}{cc}
\mathbf{0}_{1, r} & 0 \\
\mathbf{I}_{r} & \mathbf{0}_{r, 1}
\end{array}\right], \quad \mathbf{G}=\left[\begin{array}{cc}
1 & -\boldsymbol{\varphi}(1)^{\mathrm{T}} \\
\mathbf{0}_{r, 1} & \mathbf{I}_{r}
\end{array}\right],
$$

and $\mathbf{B}_{l}^{\phi}=\left(\beta_{i k}^{\phi(l)}\right) \in \mathbb{R}^{m \times r}, \Gamma_{l}^{\phi}=\left(\gamma_{i j}^{\phi(l)}\right) \in \mathbb{R}^{m \times m}, \mathbf{W}_{n}=\left(\omega_{i k}^{(n)}\right) \in \mathbb{R}^{m \times r}, \mathbf{P}_{n}=\left(\rho_{i j}^{(n)}\right) \in$ $\mathbb{R}^{m \times m}$, where

$$
\begin{aligned}
& \beta_{i k}^{\phi(l)}=\int_{0}^{1}\left(\phi\left(v_{n, i}\right)-\phi\left(v_{l}+s h\right)\right)^{-\alpha} \phi^{\prime}\left(v_{l}+s h\right) \varphi_{k}(s) \mathrm{d} s, \\
& \gamma_{i j}^{\phi(l)}=\int_{0}^{1}\left(\phi\left(v_{n, i}\right)-\phi\left(v_{l}+s h\right)\right)^{-\alpha} \phi^{\prime}\left(v_{l}+s h\right) \psi_{j}(s) \mathrm{d} s, \\
& \omega_{i k}^{(n)}=\frac{1}{h}\left(\frac{c_{n, i} h_{n}}{2}\right)^{1-\alpha} \int_{-1}^{1}(1-s)^{-\alpha} M_{n, k}\left(\frac{c_{n, i}}{2}(1+s)\right) \mathrm{d} s, \\
& \rho_{i j}^{(n)}=\frac{1}{h}\left(\frac{c_{n, i} h_{n}}{2}\right)^{1-\alpha} \int_{-1}^{1}(1-s)^{-\alpha} L_{n, j}\left(\frac{c_{n, i}}{2}(1+s)\right) \mathrm{d} s .
\end{aligned}
$$

The main result of this section is the following theorem.

Theorem 4.1. Let $z=\lambda$ h. The multistep collocation method (3.5) for the Eq. (4.1) comprises the recurrence relation

$$
\left[\begin{array}{c}
y_{n+1}^{\phi} \\
\boldsymbol{y}_{n}^{\phi(r)} \\
\mathbf{U}_{n}^{\phi}
\end{array}\right]=\mathbf{R}_{n}(z)\left[\begin{array}{c}
y_{n}^{\phi} \\
\boldsymbol{y}_{n-1}^{\phi(r)} \\
\mathbf{U}_{n-1}^{\phi}
\end{array}\right], \quad 1 \leqslant n \leqslant N-1,
$$

with the stability matrix $\mathbf{R}_{n}(z)=\left[\mathbf{Q}_{n}(z)\right]^{-1} \mathbf{M}_{n}(z)$ and

$$
\begin{aligned}
\mathbf{Q}_{n}(z) & :=\left[\begin{array}{ccc}
\mathbf{0}_{m, 1} & -z \mathbf{W}_{n} & \mathbf{I}_{m}-z \mathbf{P}_{n} \\
\mathbf{G} & \mathbf{E}
\end{array}\right], \\
\mathbf{M}_{n}(z) & :=\left[\begin{array}{ccc}
\mathbf{0}_{m, 1} & z\left(\mathbf{B}_{n-1}^{\phi}-\mathbf{W}_{n-1}\right) & \mathbf{I}_{m}+z\left(\Gamma_{n-1}^{\phi}-\mathbf{P}_{n-1}\right) \\
\mathbf{F} & \mathbf{0}_{r+1, m}
\end{array}\right] .
\end{aligned}
$$

Proof. For the Eq. (4.1), we rewrite (3.6) and (3.7) as follows

$$
\begin{aligned}
F_{n, i}^{\phi} & =1+z \sum_{l=0}^{n-1} \int_{0}^{1}\left(\phi\left(v_{n, i}\right)-\phi\left(v_{l}+s h\right)\right)^{-\alpha} \phi^{\prime}\left(v_{l}+s h\right) U_{l, s}^{\phi} \mathrm{d} s, \\
\Phi_{n, i}^{\phi} & =\frac{z}{h}\left(\frac{\phi\left(v_{n, i}\right)-\phi\left(v_{n}\right)}{2}\right)^{1-\alpha} \int_{-1}^{1}(1-s)^{-\alpha} u_{n}\left(\phi\left(v_{n}\right)+\frac{\phi\left(v_{n, i}\right)-\phi\left(v_{n}\right)}{2}(1+s)\right) \mathrm{d} s .
\end{aligned}
$$


Taking into account that

$$
\begin{aligned}
& y^{\phi}\left(v_{n, i}\right)=y_{n, i}^{\phi}=U_{n, i}^{\phi}, \\
& U_{n, s}^{\phi}=\sum_{k=0}^{r-1} \varphi_{k}(s) y_{n-k}^{\phi}+\sum_{j=1}^{m} \psi_{j}(s) y_{n, j}^{\phi},
\end{aligned}
$$

we write

$$
U_{n, i}^{\phi}=1+z \sum_{l=0}^{n-1}\left(\sum_{k=0}^{r-1} \beta_{i k}^{\phi(l)} y_{l-k}^{\phi}+\sum_{j=1}^{m} \gamma_{i j}^{\phi(l)} U_{l, j}^{\phi}\right)+z \sum_{k=0}^{r-1} \omega_{i k}^{(n)} y_{n-k}^{\phi}+z \sum_{j=1}^{m} \rho_{i j}^{(n)} U_{n, j}^{\phi} .
$$

Now the multistep collocation method (3.5) can be represented in a more compact form viz.

$$
\begin{aligned}
y_{n+1}^{\phi} & =\varphi(1)^{\mathrm{T}} \boldsymbol{y}_{n}^{\phi(r)}+\boldsymbol{\psi}(1)^{\mathrm{T}} \mathbf{U}_{n}^{\phi}, \\
\mathbf{U}_{n}^{\phi} & =\mathbf{u}+z \sum_{l=0}^{n-1}\left(\mathbf{B}_{l}^{\phi} \boldsymbol{y}_{l}^{\phi(r)}+\boldsymbol{\Gamma}_{l}^{\phi} \mathbf{U}_{l}^{\phi}\right)+z \mathbf{W}_{n} \boldsymbol{y}_{n}^{\phi(r)}+z \mathbf{P}_{n} \mathbf{U}_{n}^{\phi} .
\end{aligned}
$$

Considering the difference $\mathbf{U}_{n}^{\phi}-\mathbf{U}_{n-1}^{\phi}$, we find out

$$
\begin{aligned}
\mathbf{U}_{n}^{\phi}-\mathbf{U}_{n-1}^{\phi}= & z\left(\mathbf{B}_{n-1}^{\phi} \boldsymbol{y}_{n-1}^{\phi(r)}+\Gamma_{n-1}^{\phi} \mathbf{U}_{n-1}^{\phi}\right)+z \mathbf{W}_{n} \boldsymbol{y}_{n}^{\phi(r)}-z \mathbf{W}_{n-1} \boldsymbol{y}_{n-1}^{\phi(r)} \\
& +z \mathbf{P}_{n} \mathbf{U}_{n}^{\phi}-z \mathbf{P}_{n-1} \mathbf{U}_{n-1}^{\phi} .
\end{aligned}
$$

Therefore,

$$
\begin{aligned}
\left(\mathbf{I}_{m}-z \mathbf{P}_{n}\right) \mathbf{U}_{n}^{\phi}-z \mathbf{W}_{n} \boldsymbol{y}_{n}^{\phi(r)}= & z\left(\mathbf{B}_{n-1}^{\phi}-\mathbf{W}_{n-1}\right) \boldsymbol{y}_{n-1}^{\phi(r)} \\
& +\left[\mathbf{I}_{m}+z\left(\Gamma_{n-1}^{\phi}-\mathbf{P}_{n-1}\right)\right] \mathbf{U}_{n-1}^{\phi} .
\end{aligned}
$$

It follows from (4.3) that

$$
\left[\begin{array}{cc}
1 & -\varphi(1)^{\mathrm{T}} \\
\mathbf{0}_{r, 1} & \mathbf{I}_{r}
\end{array}\right]\left[\begin{array}{c}
y_{n+1}^{\phi} \\
\boldsymbol{y}_{n}^{\phi(r)}
\end{array}\right]+\left[\begin{array}{c}
-\boldsymbol{\psi}(1)^{\mathrm{T}} \\
\mathbf{0}_{r, m}
\end{array}\right] \mathbf{U}_{n}^{\phi}=\left[\begin{array}{cc}
\mathbf{0}_{1, r} & 0 \\
\mathbf{I}_{r} & \mathbf{0}_{r, 1}
\end{array}\right]\left[\begin{array}{c}
y_{n}^{\phi} \\
\boldsymbol{y}_{n-1}^{\phi(r)}
\end{array}\right] .
$$

Taking into account the Eq. (4.5), we obtain

$$
\begin{aligned}
& {\left[\begin{array}{ccc}
\mathbf{0}_{m, 1} & -z \mathbf{W}_{n} & \mathbf{I}_{m}-z \mathbf{P}_{n} \\
& \mathbf{G} & \mathbf{E}
\end{array}\right]\left[\begin{array}{c}
y_{n+1}^{\phi} \\
\boldsymbol{y}_{n}^{\phi(r)} \\
\mathbf{U}_{n}^{\phi}
\end{array}\right] } \\
= & {\left[\begin{array}{ccc}
\mathbf{0}_{m, 1} & z\left(\mathbf{B}_{n-1}^{\phi}-\mathbf{W}_{n-1}\right) & \mathbf{I}_{m}+z\left(\boldsymbol{\Gamma}_{n-1}^{\phi}-\mathbf{P}_{n-1}\right) \\
& \mathbf{F} & \mathbf{0}_{r+1, m}
\end{array}\right]\left[\begin{array}{c}
y_{n}^{\phi} \\
y_{n-1}^{\phi(r)} \\
\mathbf{U}_{n-1}^{\phi}
\end{array}\right], }
\end{aligned}
$$

which implies the representation (4.2). 


\section{Numerical Experiments}

Usually, the integrals in the Eqs. (3.6) and (3.7) cannot be evaluated analytically, hence quadrature formulae have to be used. The integral in the right-hand side of (3.6) does not contain singular terms. Therefore, one can use the Gauss-Legendre quadratures thus obtaining

$$
\bar{F}_{n, i}^{\phi}=g^{\phi}\left(v_{n, i}\right)+h \sum_{l=0}^{n-1} \sum_{\eta=0}^{\mu_{1}} b_{\eta} K^{\phi}\left(v_{n, i}, v_{l}+\xi_{\eta} h\right) u_{l}^{\phi}\left(v_{l}+\xi_{\eta} h\right),
$$

where $\left(\xi_{\eta}, b_{\eta}\right)_{\eta=0}^{\mu_{1}}$ are Gauss-Legendre nodes and weights in $[0,1]$.

To discretise the term (3.7), we approximate it by Gauss-Jacobi quadrature formula, so that

$$
\bar{\Phi}_{n, i}^{\phi}=\left(\frac{c_{n, i} h_{n}}{2}\right)^{1-\alpha} \sum_{\eta=0}^{\mu_{0}} d_{\eta} k\left(t_{n, i}, t_{n}+\frac{c_{n, i} h_{n}}{2}\left(1+\gamma_{\eta}\right)\right) u_{n}\left(t_{n}+\frac{c_{n, i}}{2}\left(1+\gamma_{\eta}\right) h_{n}\right),
$$

where $\left(\gamma_{\eta}, d_{\eta}\right)_{\eta=0}^{\mu_{0}}$ are Gauss-Jacobi nodes and weights in $[-1,1]$.

The starting values of the method are determined by the classical one-step collocation method of order $m+r$, and the discretised multistep collocation polynomial $P_{n}^{\phi}(v)$ has the form

$$
P_{n}^{\phi}\left(v_{n}+s h\right)=\sum_{k=0}^{r-1} \varphi_{k}(s) y_{n-k}^{\phi}+\sum_{j=1}^{m} \psi_{j}(s) Y_{n, j}^{\phi}, \quad s \in[0,1], \quad n=0,1, \cdots, N-1 .
$$

The second collocation polynomial has the form

$$
P_{n}\left(t_{n}+s h_{n}\right)=\sum_{k=0}^{r-1} M_{n, k}(s) y_{n-k}+\sum_{j=1}^{m} L_{n, j}(s) Y_{n, j}, \quad s \in[0,1], \quad n=0,1, \cdots, N-1 .
$$

Thus, the Eqs. (5.3) and (5.4) represent the continuous approximations of $u_{l}^{\phi}$ and $u_{l}$, respectively. Besides, $Y_{n, j}^{\phi}=Y_{n, j}=P_{n}^{\phi}\left(v_{n, j}\right)=P_{n}\left(t_{n, j}\right)$ are determined by the nonlinear system

$$
\begin{aligned}
& Y_{n, i}^{\phi}=\bar{F}_{n, i}^{\phi}+\bar{\Phi}_{n, i}^{\phi}, \quad i=1,2, \cdots, m, \\
& y_{n+1}^{\phi}=\sum_{k=0}^{r-1} \varphi_{k}(1) y_{n-k}^{\phi}+\sum_{j=1}^{m} \psi_{j}(1) Y_{n, j}^{\phi} .
\end{aligned}
$$

In order to verify the theoretical results of Section 3, we consider two test problems.

Example 5.1. The linear weakly singular Volterra integral equation

$$
y(t)=\frac{1}{2} \pi t+t^{\frac{1}{2}}-\int_{0}^{t}(t-s)^{-1 / 2} y(s) \mathrm{d} s, \quad t \in[0,1]
$$

has the exact solution $y(t)=t^{1 / 2}-\mathrm{cf}$. [26, Example 1]. 
Table 2: Example 5.1: Error Norms and Order of Convergence.

\begin{tabular}{||ccccc||}
\hline \multicolumn{4}{||c}{$c_{1}=21 / 38, c_{2}=1$} & \multicolumn{2}{c||}{$c_{1}=16 / 19, c_{2}=1$} \\
\hline$N$ & Error Norm & Order & Error Norm & Order \\
\hline 4 & $1.0336 \mathrm{e}-003$ & - & $9.3550 \mathrm{e}-004$ & - \\
8 & $4.6645 \mathrm{e}-005$ & 4.4698 & $4.2028 \mathrm{e}-005$ & 4.4763 \\
16 & $1.8953 \mathrm{e}-006$ & 4.6212 & $1.6840 \mathrm{e}-006$ & 4.6414 \\
32 & $8.3248 \mathrm{e}-008$ & 4.5089 & $7.3189 \mathrm{e}-008$ & 4.5241 \\
64 & $3.5540 \mathrm{e}-009$ & 4.5499 & $3.1229 \mathrm{e}-009$ & 4.5507 \\
128 & $1.1492 \mathrm{e}-010$ & 4.9507 & $1.0225 \mathrm{e}-010$ & 4.9327 \\
256 & $5.0575 \mathrm{e}-012$ & 4.5061 & $4.3201 \mathrm{e}-012$ & 4.5649 \\
512 & $2.2371 \mathrm{e}-013$ & 4.4987 & $1.8433 \mathrm{e}-013$ & 4.5507 \\
1024 & $9.9198 \mathrm{e}-015$ & 4.4987 & $7.8650 \mathrm{e}-015$ & 4.5507 \\
\hline
\end{tabular}

We set $m=2, r=3$ with $c_{1}=21 / 38, c_{2}=1$ and $c_{1}=16 / 19, c_{2}=1$, respectively, and use a smoothing transformation $\phi(v)=T V^{-d} v^{d}, 0 \leqslant v \leqslant V$, which satisfy the conditions (2.2)-(2.4). Taking into account [23, Theorems 5.2], we obtain $d>4$, and we will use the transformation $t=\phi(v)=v^{7}$. The starting values are obtained by the classical one-step collocation method of order 5 considered in [23]. Besides, we choose $\mu_{0}=\mu_{1}=3$ in the quadrature methods (5.1) and (5.2).

The errors of the method are presented in Table 2. The column "Error Norm" demonstrates absolute errors at the end point for various $N$. The column "Order" shows the estimated order of convergence computed as Order $=\log _{2}(\operatorname{Error}(N / 2) / \operatorname{Error}(N))$. For $\alpha=1 / 2$, the theoretical convergence order determined by Theorem 3.2 is $m+r-\alpha=$ $3+2-1 / 2=9 / 2$ consistent with the numerical results in Table 2 .

Example 5.2. The nonlinear weakly singular Volterra integral equation

$$
y(t)=t^{1 / 3}\left(1-\frac{9}{10} t^{4 / 3}\right)+\int_{0}^{t}(t-s)^{-1 / 3} y^{3}(s) \mathrm{d} s, \quad t \in[0,1]
$$

has the exact solution $y(t)=t^{1 / 3}-\mathrm{cf}$. [21, Example 4].

We set $m=2, r=3$ with $c_{1}=16 / 19, c_{2}=1$ and take the smoothing transformation $\phi(v)=v^{11}$. For $\alpha=1 / 3$, the theoretical convergence order determined by Theorem 3.2 is $m+r-\alpha=2+3-1 / 3 \approx 4.6667$. Table 3 shows the errors and the corresponding convergence order for the methods presented here and in [21]. We point out that the above multistep collocation methods nearly attains the theoretical value of Theorem 3.2, while the method in [21] produces visibly distinct results.

\section{Acknowledgments}

The authors are greatly indebted to the referees for useful comments. Particular thanks go to Professor Victor Didenko who made numerous helpful suggestions. This work was supported by the National Natural Science Foundation of China under the Grants Nos. 11771112 and 11671112. 
Table 3: Example 5.2: Two methods.

\begin{tabular}{||ccccc||}
\hline \multicolumn{4}{||c}{ Our method } & \multicolumn{2}{c||}{ Method in [21] } \\
\hline$N$ & Error Norm & Order & Error Norm & Order \\
\hline 4 & $8.9885 \mathrm{e}-004$ & - & $1.7538 \mathrm{e}-002$ & - \\
8 & $3.8011 \mathrm{e}-005$ & 4.5636 & $9.6438 \mathrm{e}-003$ & 0.8628 \\
16 & $1.6001 \mathrm{e}-006$ & 4.5702 & $2.8406 \mathrm{e}-003$ & 1.7634 \\
32 & $6.4019 \mathrm{e}-008$ & 4.6435 & $6.1128 \mathrm{e}-004$ & 2.2163 \\
64 & $2.6456 \mathrm{e}-009$ & 4.5968 & $1.3058 \mathrm{e}-004$ & 2.2269 \\
128 & $1.0679 \mathrm{e}-010$ & 4.6308 & $2.7371 \mathrm{e}-005$ & 2.2542 \\
256 & $4.3684 \mathrm{e}-012$ & 4.6115 & $5.7373 \mathrm{e}-006$ & 2.2542 \\
512 & $1.7870 \mathrm{e}-013$ & 4.6115 & $1.2026 \mathrm{e}-006$ & 2.2542 \\
1024 & $7.3101 \mathrm{e}-015$ & 4.6115 & $2.5209 \mathrm{e}-007$ & 2.2542 \\
\hline
\end{tabular}

\section{References}

[1] J. Abdalkhani, A modified approach to the numerical solution of linear weakly singular Volterra integral equations of the second kind, J. Integral Equations Appl. 5, 149-166 (1993).

[2] P. Baratella and A.P. Orsi, A new approach to the numerical solution of weakly singular Volterra integral equations, J. Comput. Appl. Math. 163, 401-418 (2004).

[3] H. Brunner, The numerical solution of weakly singular Volterra integral equations by collocation on graded meshes, Math. Comput. 45, 417-437 (1985).

[4] H. Brunner, The approximate solution of Volterra equations with nonsmooth solutions, Utilitas Math. 27, 57-95 (1985).

[5] H. Brunner, Collocation Methods for Volterra Integral and Related Functional Equations, Cambridge University Press (2004).

[6] H. Brunner, M.R. Crisci, E. Russo and A. Vecchio, A family of methods for Abel integral equations of the second kind, J. Comput. Appl. Math. 34, 211-219 (1991).

[7] H. Brunner and P.J. van der Houwen, The Numerical Solutions of Volterra Equations, NorthHolland Publishing Company (1986).

[8] H. Brunner, A. Pedas and G. Vainikko, The piecewise polynomial collocation method for nonlinear weakly singular Volterra equations, Math. Comput. 68, 1079-1095 (1999).

[9] A. Cardone, D. Conte and B. Paternoster, A family of multistep collocation methods for Volterra integro-differential equations, AIP Conf. Proc. 1168, 358-361 (2009).

[10] Y. Chen and T. Tang, Spectral methods for weakly singular Volterra integral equations with smooth solutions, J. Comput. Appl. Math. 233, 938-950 (2009).

[11] D. Conte, Z. Jackiewicz and B. Paternoster, Two-step almost collocation methods for Volterra integral equations, Appl. Math. Comput. 204, 839-853 (2008).

[12] D. Conte and B. Paternoster, Multistep collocation methods for Volterra integral equations, Appl. Numer. Math. 59, 1721-1736 (2009).

[13] P.J. Davis, Interpolation and Approximation, Dover Publications (1975).

[14] T. Diogo, P.M. Lima, A. Pedas and G. Vainikko, Smoothing transformation and spline collocation for weakly singular Volterra integro-differential equations, Appl. Numer. Math. 114, 63-76 (2017).

[15] T. Diogo, J. Ma and M. Rebelo, Fully discretized collocation methods for nonlinear singular Volterra integral equations, J. Comput. Appl. Math. 247, 84-101 (2013). 
[16] T. Diogo, S. McKee and T. Tang, Collocation methods for second-kind Volterra integral equations with weakly singular kernels, Proc. Royal Soc. Edinburgh Sect. A 124, 199-210 (1994).

[17] E.A. Galperin, E.J. Kansa, A. Makroglou and S.A. Nelson, Variable transformations in the numerical solution of second kind Volterra integral equations with continuous and weakly singular kernels; extensions to Fredholm integral equations, J. Comput. Appl. Math. 115, 193-211 (2000).

[18] R. Garrappa, Order conditions for Volterra Runge-Kutta methods, Appl. Numer. Math. 60, 561573 (2010).

[19] F.R. de Hoog and R. Weiss, High order methods for a class of Volterra integral equations with weakly singular kernels, SIAM J. Numer. Anal. 11, 1166-1180 (1974).

[20] G. Izzo, Z. Jackiewicz, E. Messina and A. Vecchio, General linear methods for Volterra integral equations, J. Comput. Appl. Math. 234, 2768-2782 (2010).

[21] D. Nazari Susahab and S. Shahmorad, Multistep collocation methods for weakly singular Volterra integral equations with application to fractional differential equations, http://cn. arxiv.org/abs/1408.4029.

[22] J. Norbury and A.M. Stuart, Volterra integral equations and a new Gronwall inequality, Part I: The linear case, Proc. Royal Soc. Edinburgh Sect. A 106, 361-373 (1987).

[23] A. Pedas and G. Vainikko, Smoothing transformation and piecewise polynomial collocation for weakly singular Volterra integral equations, Computing 73, 271-293 (2004).

[24] I. Podlubny, Fractional Differential Equations, Academic Press (1999).

[25] H.J.J. te Riele, Collocation methods for weakly singular second-kind Volterra integral equations with non-smooth solution, IMA J. Numer. Anal. 2, 437-449 (1982).

[26] S. Sohrabi, H. Ranjbar and M. Saei, Convergence analysis of the Jacobi-collocation method for nonlinear weakly singular Volterra integral equations, Appl. Math. Comput. 299, 141-152 (2017).

[27] C. Zhang and S. Vandewalle, General linear methods for Volterra integro-differential equations with memory, SIAM J. Sci. Comput. 27, 2010-2031 (2006). 